\title{
Unit of Entropy
}

National Cancer Institute

\section{Source}

National Cancer Institute. Unit of Entropy. NCI Thesaurus. Code C70446.

A unit used to express the number of microscopic configurations resulting in the observed macroscopic description of the thermodynamic system that represents the amount of uncertainty which remains about a system, after its observable macroscopic properties have been taken into account. 